\title{
Agreement on responses to respiratory illnesses questionnaire
}

\author{
Tze Wai Wong, Tak Sun Yu, Joseph Lip Yiu Liu, Siu Lan Wong
}

\begin{abstract}
The prevalence of respiratory symptoms and illnesses among 2225 schoolchildren in Hong Kong was studied by questionnaires administered independently to them and their parents. The agreement was generally poor for respiratory symptoms. The disparity shows the need for cross validation of clinical information in history taking.

(Arch Dis Child 1998;78:379-380)
\end{abstract}

Keywords: questionnaires; respiratory illness

In paediatric practice, clinical information is usually sought from both children and their parents. Knowledge of the reliability of the two sources of information can assist the clinician in assessing the quality of evidence obtained in a clinical consultation. A recent study of South African children showed good agreement of responses from two data collection methods for asthma symptoms using two methods of questionnaire administration. ${ }^{1}$ In the present study we aimed to determine the level of agreement to responses on common respiratory symptoms and illnesses in Hong Kong schoolchildren from two sets of respondents: the children and their parents.

\section{Methods}

The target population consisted of 8 to 12 year old schoolchildren in three districts of Hong Kong. Four schools from each district were randomly selected to participate in the study, using two stage cluster sampling. The study was carried out between February and April 1995. Data on the children's prevalence of four respiratory symptoms (cough, phlegm, wheezing, and shortness of breath) in the past three months, and lifetime prevalence of six respiratory illnesses (asthma, allergic rhinitis, sinusitis, pneumonia, bronchitis, and tuberculosis, as diagnosed by their doctors) were obtained through questionnaires completed by the schoolchildren and their parents. Both parents were asked to complete their questionnaire jointly at home. The children's questionnaires were self administered in their classrooms, under the supervision of a team of research workers using a standard set of procedures. For the diagnosed illnesses, the parents were asked whether their children were ever labelled by a physician as having the illnesses. The questionnaire (in Chinese) was adapted from the American Thoracic Society questionnaires.

The prevalence rates of the respiratory symptoms and illnesses as reported by the children and their parents were calculated. The overall proportion of agreement between the two methods of data collection was calculated as the total number of subjects with identical responses reported by both methods divided by the total number of subjects. Estimates of Cohen's $\kappa$ were computed as outlined by Fleiss. $^{2}$ The interpretation of the $\kappa$ is as follows: $\kappa=1$ if there is complete agreement

$\kappa \geqslant 0$ if the observed agreement is greater than or equal to chance agreement

$\kappa \leqslant 0$ if the observed agreement is less than or equal to chance agreement.

According to Landis and Koch, ${ }^{3}$ a $\kappa$ value $>0.75$ implies excellent agreement beyond chance between the two methods. Values $\geqslant 0.4$ and $\leqslant 0.75$ denote fair to good agreement beyond chance. Values $<0.4$ denote poor agreement. Data were analysed using SPSS for Windows 6. 1.

\section{Results}

All 12 schools which were sampled agreed to take part in the study. In all, 2292 children completed the questionnaires in class (response rate $86 \%$ ). Of these children, 2225 $(97 \%)$ returned their parents' questionnaires: 1061 were aged 8 to 10 years, and 1164 were from 10 to 12 years. The prevalences, overall proportions of agreement, and $\kappa$ statistics of the respiratory symptoms and illnesses are shown in table 1 . The prevalence of cough, phlegm, and wheezing, and of pneumonia and bronchitis as reported by the parents were significantly higher than those reported by their children, while the reverse was found for shortness of breath. Prevalences of asthma, allergic rhinitis, sinusitis, and tuberculosis reported by the children were similar to the corresponding rates obtained independently by the parents. The proportions of agreement ranged from $71.3 \%$ to $91.2 \%$ for the four symptoms and from $85.0 \%$ to $99.6 \%$ for the six illnesses. By Landis and Koch's criteria for $\kappa{ }^{3}$ the agreement was generally poor for the respiratory symptoms and tuberculosis, fair for sinusitis, bronchitis and pneumonia, and good for allergic rhinitis and asthma. When we compared the agreement by age group, significantly higher values were observed among children aged 10 to 12 years for the following symptoms or illnesses: wheezing, shortness of breath, asthma, allergic rhinitis, and sinusitis.

\section{Discussion}

Recent studies on the reliability of respiratory health questionnaires have concentrated on the reproducibility of parents' responses to questions on asthma or respiratory symptoms in their children, using different modes of ques- 
Table 1 Agreement between responses to questions on childhood respiratory illness in questionnaires completed by children and those completed by parents

\begin{tabular}{|c|c|c|c|c|c|c|c|c|c|c|}
\hline & \multicolumn{4}{|c|}{ No of subjects* } & \multirow{3}{*}{$\begin{array}{l}\text { Prevalence } \\
\text { reported by } \\
\text { children (\%) }\end{array}$} & \multirow{3}{*}{$\begin{array}{l}\text { Prevalence } \\
\text { reported by } \\
\text { parents (\%) }\end{array}$} & \multirow{3}{*}{$\begin{array}{l}\text { Proportion } \\
\text { observed } \\
\text { agreement (\%) }\end{array}$} & \multirow{2}{*}{\multicolumn{3}{|c|}{$\kappa$ Statistic }} \\
\hline & Parents + & Parents+ & Parents- & Parents- & & & & & & \\
\hline & Child + & Child- & Child+ & Child- & & & & All ages & 8-9 years & $10-12$ years \\
\hline \multicolumn{11}{|c|}{ Respiratory symptom } \\
\hline Cough & 293 & 281 & 173 & 1413 & 21.6 & 26.4 & 79.0 & 0.43 & 0.44 & 0.42 \\
\hline Phlegm & 355 & 371 & 240 & 1159 & 28.0 & 32.9 & 71.3 & 0.33 & 0.32 & 0.34 \\
\hline Wheeze & 65 & 117 & 83 & 1776 & 7.3 & 8.9 & 90.2 & 0.34 & 0.26 & 0.41 \\
\hline Short of breath & 70 & 60 & 113 & 1727 & 9.3 & 6.6 & 91.2 & 0.40 & 0.31 & 0.48 \\
\hline \multicolumn{11}{|l|}{ Respiratory disease } \\
\hline Asthma & 89 & 23 & 41 & 1667 & 7.1 & 6.2 & 96.5 & 0.72 & 0.65 & 0.76 \\
\hline Allergic rhinitis & 427 & 151 & 134 & 1188 & 29.5 & 30.4 & 85.0 & 0.64 & 0.60 & 0.68 \\
\hline Sinusitis & 28 & 25 & 32 & 1721 & 3.3 & 2.9 & 96.7 & 0.48 & 0.34 & 0.57 \\
\hline Pneumonia & 59 & 61 & 19 & 1688 & 4.3 & 6.6 & 95.6 & 0.57 & 0.60 & 0.54 \\
\hline Bronchitis & 164 & 177 & 70 & 1451 & 12.6 & 18.3 & 86.7 & 0.50 & 0.50 & 0.50 \\
\hline Tuberculosis & 2 & 1 & 6 & 1788 & 0.4 & 0.2 & 99.6 & 0.36 & 0.67 & 0.25 \\
\hline
\end{tabular}

${ }^{\star}$ Refers to number of children with/without self reported (child $+/-$ ) or parent reported (parents $+/-$ ) symptoms or diseases. The total number of responses was less than 2225 because of incomplete data in some questionnaires.

tionnaire administration (self completed and interviewer administered) on different occasions, with certain symptoms found to be more repeatable than others. ${ }^{45}$ Our study focused on recent respiratory symptoms and the lifetime prevalence of common doctor diagnosed respiratory illnesses among 8 to 12 year old schoolchildren in Hong Kong. It should be noted that the parental questionnaires have been assumed to be completed jointly by the natural parents, which we did not verify. Also, in the absence of a gold standard, data from the two sources (children and their parents) provide no information regarding their validity. The proportions of agreement for respiratory symptoms and illnesses were uniformly high. However, this parameter was influenced by the large numbers of concordant negative responses for diseases of low prevalence, such as tuberculosis (with $99.6 \%$ agreement). The $\kappa$ statistic, which corrects for chance, is a better indicator of agreement. The fair to good agreement observed for most physician diagnosed illnesses might have been due to "labelling effect". The agreement for respiratory symptoms ranged from fair to poor, in contrast to findings by Clifford et $a l^{5}$ which showed good agreement for wheeze $(\kappa=0.78)$ and fair for shortness of breath $(\kappa=0.50)$. A possible explanation is the lower prevalence of wheeze among schoolchildren in Hong Kong than in the United Kingdom. Of relevance to clinical practice is the disparity in the reporting of respiratory symptoms between parents and children, especially in children aged below 10 years of age. This indicates the need to take a clinical history from both parents and children, and to be cautious in its interpretation when the history is obtained from either source alone. When investigating childhood exposures for which parents have little knowledge, data from both parent and children may offer greater insights, and discordant results may be informative. ${ }^{6}$

1 Ehriich RI, Du Toit D, Jordaan E, et al. Prevalence and relibility of asthma symptoms in primary schoolchildren in Cape Town. Int $\mathcal{F}$ Epidemiol 1995;24:1138-46.

2 Fleiss JL. Statistical methods for rates and proportions. 2 nd Ed. New York: John Wiley, 1981:212.

3 Landis JR, Koch GG. The measurement of observer agreement for categorical data. Biometrics 1977;33:159-74.

4 Peat JK, Salome CM, Toelle BG, et al. Reliability of a respiratory history questionnaire and effect of mode of administration on classification of asthma in children. Chest 1992; 102:153-7.

5 Clifford RD, Radford M, Howell JB, et al. Prevalence of respiratory symptoms among 7 and 11 year old schoolchildren
perd al. Prevalence of resin Leicestershire. Arch Dis Child 1989; 64:1118-25.

6 Whiteman D, Green A. Wherein lies the truth? Assessment of agreement between parent proxy and child respondents. Int $\mathscr{f}$ Epidemiol 1997;26:855-9. 